\title{
Educación: medio reparador de víctimas y victimarios del conflicto armado colombiano
}

Ferney Hernán Rincón Ospina

https://orcid.org/0000-0002-0806-5015

Universidad de Antioquia, Colombia

ferney.rincon@udea.edu.co

\section{Resumen}

Este artículo presenta un análisis de la educación como puente entre víctimas y victimarios del conflicto armado colombiano, para desarrollar procesos de reparación y reconciliación entre ellos. Tanto el material textual como su análisis parten de la experiencia del autor en procesos de reconciliación a lo largo de diez años. La experiencia se presenta en cuatro partes: en la primera, se caracteriza el contexto en el que se realizó el proceso formativo; en la segunda, se hace el análisis de cuatro elementos relevantes del proceso de reparación y reconciliación entre víctimas y victimarios: encuentros terapéuticos de reconciliación, inclusión escolar, convivencia comofactor reconciliador y acompañamiento psicosocial; en la tercera parte se muestra cómo estos elementos permitieron realizar procesos reales de reparación y reconciliación entre ambos grupos poblacionales. Por último, se justifica y evidencia el papel preponderante de la educación como medio transformador en población en condición de vulnerabilidad psicosocial.

\section{Palabras clave (Fuente: tesauro de la Unesco)}

Colombia; conflicto armado; educación escolar; educación para la paz; educación para el desarme; guerra; pedagogía social; resolución de conflictos; solución de conflictos.

Recepción: 01/08/2020 | Envío a pares: 16/11/2020 | Aceptación por pares: 18/12/2020 | Aprobación: 09/02/2021 


\title{
Education: A Means to Reparation for Victims and Perpetrators of the Colombian Armed Conflict
}

\begin{abstract}
This article studies education as a bridge between victims and perpetrators of the Colombian armed conflict for reparation and reconciliation processes. The textual material and its analysis rely on the author's ten years' experience in reconciliation, comprising four parts: first, the context in which education took place is characterized. The second part discusses four relevant elements of the reparation and reconciliation processes between victims and perpetrators: therapeutic reconciliation meetings, school inclusion, coexistence as a reconciling factor, and psychosocial support. Then, it is revealed how these elements allowed for actual reparation and reconciliation between both population groups. Finally, the article justifies and underscores the preponderant role of education in transforming the psychosocially vulnerable population.
\end{abstract}

\section{Keywords (Source: Unesco thesaurus)}

Armed conflicts; Colombia; school education; peace education; disarmament education; war; social pedagogy; conflict resolution; dispute settlement. 


\title{
Educação: meio reparador de vítimas e vitimários do conflito armado colombiano
}

\author{
Resumo
}

Neste artigo, é apresentada uma análise da educação como ponte entre vítimas e vitimários do conflito armado colombiano, para desenvolver processos de reparação e reconciliação entre eles. Tanto o material textual quanto sua análise partem da experiência do autor em processos de reconciliação ao longo de dez anos. A experiência é exposta em quatro partes. Na primeira, é caracterizado o contexto no qual o processo de formação foi realizado; na segunda, é feita a análise de quatro elementos relevantes do processo de reparação e reconciliação entre vítimas e vitimários (encontros terapêuticos de reconciliação, inclusão escolar, convivência como fator reconciliador e acompanhamento psicossocial); na terceira parte, é mostrado como esses elementos permitiram realizar processos reais de reparação e reconciliação entre ambos os grupos populacionais. Por último, é justificado e evidenciado o papel preponderante da educação como meio transformador na população em condição de vulnerabilidade psicossocial.

\section{Palavras-chave (Fonte: tesauro da Unesco)}

Colômbia; conflito armado; educação escolar; educação para a paz; educação para o desarmamento; guerra; pedagogia social; resolução de conflitos. 
La educación, tal como lo plantea la UNESCO (2015), no solo debe darse en las etapas iniciales del desarrollo del individuo, sino que debe ser continua a lo largo de la vida. Como lo refieren Noguchi Guevara y Yorozu (2018) "básicamente, el aprendizaje a lo largo de la vida reposa en la integración del aprendizaje y la vida, lo que comprende actividades de aprendizaje para personas de todas las edades, en todos los contextos de la vida y mediante diversas modalidades, que en conjunto responden a una amplia gama de necesidades y exigencias relativas al aprendizaje". En este sentido, la experiencia en la cual se sustenta el presente artículo está relacionada con un proceso educativo dirigido a población adulta, inicialmente de desmovilizados del conflicto armado, y que nace del acuerdo del gobierno colombiano con los paramilitares de las Autodefensas Unidas de Colombia (AUC) en 2003, que dio lugar al inicio de las desmovilizaciones progresivas de sus estructuras y al diseño de un nuevo programa de reintegración a la vida civil, destinado a los exintegrantes de grupos paramilitares y a la continuación de la "desmovilización individual" de exguerrilleros (Centro Nacional de Memoria Histórica, 2015).

Del anterior proceso, se crea así en la ciudad de Medellín el componente de educación y capacitación, que fue uno de los ejes fundamentales de la intervención a los excombatientes y sirvió como puente entre el acompañamiento psicosocial y las opciones de empleabilidad, brindando las herramientas para la capacitación y cualificación de los participantes en su ruta hacia la consolidación de proyectos de vida que les permitieran la subsistencia propia y de sus familias en el marco de la legalidad (Cortés et al., 2011). La propuesta educativa incluyó, además de los desmovilizados, a personas denominadas víctimas del conflicto, promoviendo así un espacio, no solo de aprendizaje escolar, sino también de reconciliación entre actores directos que habían estado inmersos en una "guerra" y que empezaban a vivir nuevas experiencias desde un lugar diferente.

\section{Metodología}

Este artículo presenta una metodología de carácter cualitativo-reflexivo, asumiendo la reflexividad, de acuerdo con Hand (2003), como una estrategia que nos permite movernos flexiblemente en el dinámico proceso de investigación cualitativa, con la seguridad de que no vamos a perder el rumbo. Se da, por lo tanto, un proceso de sistematización impulsado por una experiencia social educativa que busca comprender tanto el contexto como los factores o elementos en los que se enmarca. En este sentido, se recurrió a varias estrategias, lo que permitió captar la información pertinente de la cual se nutre el presente artículo. La primera ha sido la participación directa de mi parte, como autor del artículo, durante más de 10 años en el proyecto donde se desarrolló la experiencia educativa, desde el lugar de pedagogo con una actuación directa como docente y también desde el quehacer psicológico acompañando y ejecutando acciones de apoyo al proceso de formación, procurando mayor integralidad para con los estudiantes participantes. Cabe resaltar que a lo largo del proceso se fueron consolidando informes y planeaciones de las actividades y que a partir de allí se hace un proceso de sistematización.

La segunda estrategia consistió en la realización de entrevistas personales con profesionales, quienes participaron en el proyecto y tenían en su haber conocimiento directo del proceso formativo llevado a cabo con los participantes. Estos profesionales y algunos de sus comentarios se mencionan y se referencian a lo largo del artículo. Por último, se recurrió a la revisión bibliográfica de información ya sistematizada, en especial, a la realizada en el año 2011 por la Secretaría de Gobierno Alcaldía de Medellín, titulada: Centro de formación para la paz y la reconciliación CEPAR: Un aporte a la paz desde la educación y la convivencia. De este se retoman algunos datos puntuales con comentarios tanto de sus autores como de estudiantes que allí se reportan. Dicho trabajo, al igual que sus autores, se reseñan en el artículo. 
Como lo proponen Cendales y Torres (2006), la sistematización produce, principalmente, nuevas lecturas, nuevos sentidos sobre la práctica. Si bien es cierto que se basa en la voz y la mirada de sus protagonistas, el resultado es una mirada más densa y profunda de la experiencia común, de la cual puedan derivarse pistas para potenciarla o transformarla.

\section{Contextualización}

A partir del año 2002, el Estado colombiano inició un proceso de negociación con grupos denominados Autodefensas Unidas de Colombia (AUC), que terminó con la desmovilización colectiva y con la invitación abierta a grupos guerrilleros para desmovilizarse también dentro del marco de justicia transicional'. La desmovilización colectiva se dio con las $A \cup C^{2}$, lo que abrió una puerta esperanzadora para emprender una ruta diferente como sociedad. Por aquel entonces, no se dieron negociaciones colectivas con grupos insurgentes de guerrilla; sin embargo, aquellos excombatientes que por cuenta propia desertaban de estos grupos armados o eran capturados por la fuerza pública eran incluidos en el proceso de reincorporación a la vida civil, si así lo deseaban.

En la ciudad de Medellín se dieron las desmovilizaciones colectivas de AUC por parte de dos

1 Según la Sentencia C-052 de la Corte Constitucional de Colombia, "la justicia transicional es una institución jurídica de carácter excepcional, en comparación con las instituciones penales ordinarias, que tiene como piedra angular a la víctima del conflicto armado, importando más su reparación y la consecución de la verdad que el castigo al perpetrador de las violaciones. Se concentra en una justicia más restaurativa que retributiva, promoviendo la reconstruc ción del tejido social y la reconciliación de la sociedad". M.P. Nilson Pinilla Pinilla. La justicia transicional es un modelo de justicia que se adopta para garantizar los derechos de las víctimas y para que la sociedad logre la paz, la verdad, la justicia, junto con la reparación y las garantías de no repetición en los contextos de transición de un estado de violencia y de conflicto a uno de paz. Véase Bolaños y Biel (2019).

2 Según el Observatorio de Procesos de Desarme, Desmovilización y Reintegración (ODDR, 2010) de la Universidad Nacional de Colombia, durante el periodo 2003-2006, en el marco del proceso de paz entre el Gobierno Nacional y las Autodefensas, 34 estructuras de estas organizaciones se desmovilizaron colectivamente en 37 ceremonias en diferentes zonas del país. bloques: Cacique Nutibara, en noviembre de 2003, y Héroes de Granada, en agosto de 2005 (Alonso y Valencia, 2008). Ante ello, la Alcaldía Municipal creó un modelo de acompañamiento para los desmovilizados, llamado Regreso a la Legalidad, implementado a través del programa Paz y Reconciliación (PNUD, 2006). Este programa inició a partir de enero de 2004, dando atención integral y seguimiento al proceso de regreso a la legalidad de personas desmovilizadas de grupos al margen de la ley que se encontraban ubicadas en Medellín y el valle de Aburrá.

En octubre del año 2005 se crea una estrategia de acompañamiento educativo. Llamada Centro de Formación para la Paz y la Reconciliación (CEPAR), esta nace como una respuesta a la necesidad de mejorar el perfil educativo de los primeros desmovilizados atendidos en Medellín por el Programa Paz y Reconciliación, luego de haber fracasado el primer intento de vincularlos laboralmente y de iniciarlos en cursos del Servicio Nacional de Aprendizaje (SENA) (Cortés et al., 2011).

El CEPAR acogió inicialmente desmovilizados de las autodefensas y fue incorporando también a excombatientes de grupos insurgentes de guerrilla, de modo que ambos grupos poblacionales, ya como civiles, estudiaban juntos en un mismo espacio físico. También fue incluido en este proceso educativo un grupo de víctimas ${ }^{3}$ del conflicto, reconocido en la ciudad de Medellín, que conforma lo que se conoce como La Asociación Caminos de Esperanza Madres de la Candelaria4. Previo a ser incluido este grupo de

3 La Ley 1448 de 2011, llamada "Ley de víctimas y restitución de tierras", establece en su artículo 3 que se considera víctimas aquellas personas que individual o colectivamente hayan sufrido un daño por hechos ocurridos a partir del 1 de enero de 1985 , como consecuencia de infracciones al Derecho Internacional Humanitario o de violaciones graves y manifiestas a las normas internacionales de derechos humanos, ocurridas con ocasión del conflicto armado interno.

4 Caminos de Esperanza Madres de la Candelaria es una organización sin ánimo de lucro fundada en el año de 1999, con personería jurídica 21-008254-28, como respuesta a las numerosas desapariciones forzadas, secuestros y homicidios en el marco del conflicto armado colombiano. 
víctimas, se generaron interrogantes sobre la conveniencia o no de dicha decisión, ya que implicaba reunir en un mismo espacio de formación, por una parte, a desmovilizados de los grupos armados que, aunque estaban en un proceso de reintegración a la vida civil en cumplimiento de unos acuerdos pactados con el gobierno, seguían representando para buena parte de la sociedad el rol de "victimarios"s, y por otra, las víctimas, que cargaban el dolor que habían producido en ellas los múltiples crímenes de muertes y desapariciones de sus seres cercanos.

A pesar de los temores y de las preguntas sin respuestas claras, se analizaron estrategias que pudieran llevar a la materialización de la idea de vincular a las víctimas en dicho espacio formativo. En palabras de Mario Velázquez ${ }^{6}$, a quien se le realizó una entrevista personal:

Se realizó un contacto con la directora del programa de victimas de la alcaldía de Medellín para enterarla de la propuesta y en conjunto se construyó una prueba piloto que consistía en vincular a un grupo pertenecientes a la Asociación Madres de la Candelaria al proceso educativo del CEPAR; sin embargo se decidió que dicho grupo no fuera nombrado inicialmente como victimas y además eran atendidas en grupos por aparte sin tener contacto con los excombatientes, por lo menos en un primer momento.

Así fue como se dio marcha a este proceso de convergencia de estos grupos poblacionales, que representó un enorme reto para la educación en un escenario difícil por sus condiciones.

5 Si bien este término puede ser usado para referirse a cualquier persona responsable de cometer un delito, está generalmente relacionado con los procesos de paz, en donde es utilizado frecuentemente en forma plural, para referirse a los actores armados de un país, en un régimen dictatorial o democrático o en un conflicto armado interno, que han cometido actos terroristas, crímenes de guerra o crímenes de lesa humanidad.

6 M. Velásquez. Coordinador Área de Educación del Programa Paz y Reconciliación 2004-2007, entrevista personal por vía telefónica, junio de 2020 .

\section{Elementos de análisis}

\section{Encuentros terapéuticos de reconciliación}

Una forma, tal vez la más importante, de generar espacios de reparación y reconciliación entre víctimas y victimarios dentro de este contexto educativo fue haber propiciado momentos donde ambos grupos poblacionales tuvieran la oportunidad de mirarse, escucharse y comunicarse de forma directa, pero a la luz de la reflexión y con la mira puesta en reconciliarse. Cuando los sujetos perdonan, sus comportamientos (pensamientos, sentimientos, acciones) hacia quien es objeto del perdón se transforman en más positivos (Casullo, 2005). Sin lugar a dudas este tipo de eventos -que por supuesto estuvieron acompañados por profesionales que hicieron parte del centro educativo en sus diferentes roles de directivos, docentes, equipo psicosocial, entre otrosconllevaron procesos de reparación a partir del reconocimiento del lugar que ha ocupado cada uno dentro del conflicto.

El primer encuentro, descrito anteriormente, se convirtió en el punto de partida para establecer este mecanismo como una estrategia exitosa y a la vez necesaria. Por lo tanto, debía darse de forma paulatina en otros momentos dentro del cepar. Para ello, fue necesario establecer encuentros previos de sensibilización y de preparación con cada grupo poblacional con el apoyo de equipo psicosocial, buscando analizar diversas variables a la luz de la experiencia vivida como victimario y como víctima, con todas las perspectivas de orden subjetivo que dicho lugar o rol asigna a sus protagonistas.

En estos encuentros se realizaba una sensibilización previa, teniendo en cuenta la importancia de reconocimiento del daño causado a otros a partir de su estancia en el grupo armado, pero además el reto de reparar, de manera simbólica, a sus propias víctimas, lo que implicaba reunirse con ellas y ofrecerles su disposición para aceptar y enmendar sus faltas. Por otro lado, el grupo de estudiantes víctimas contó con el apoyo psicosocial de profesiona- 
les pertenecientes al programa de víctimas de la alcaldía de Medellín, quienes por medio de un trabajo grupal hicieron una preparación previa al encuentro con los victimarios; así lo relata Mario Velásquez:

Se hizo un ejercicio de preparación previa con cada grupo por separado, se les dijo a los excombatientes que acá había un grupo de víctimas, de igual manera desde el equipo psicosocial del componente de víctimas se llevó a cabo un proceso de sensibilización [...]; una vez realizado esto se hizo un evento durante una tarde en el que se reunieron ambos grupos y se hizo un trabajo grupal con ellos, se hicieron algunos ejercicios de perdón, algunos se reconocieron tanto en su rol de víctima como de victimario en su momento, luego de esto todo fue marchando de una forma armónica, de una forma normal.

Una vez dadas las condiciones, se dio el encuentro entre ambos grupos, el cual, como lo expresa Mario Velázquez, fue exitoso: "hubo en el encuentro un ejercicio de perdón y de reconocimiento, y de ahí en adelante todo fue marchando de manera armónica en el proceso de formación".

Este fue el primer grupo terapéutico sobre perdón y reconciliación. Posterior a ello, se llevaron a cabo otros encuentros, en los cuales eran seleccionadas algunas personas de cada grupo, de forma voluntaria, para que participaran directamente en el evento. Los victimarios debían expresar de manera abierta su responsabilidad dentro del conflicto y los posibles daños causados a las personas entregando una vela encendida a una de las víctimas y pidiéndole perdón. Por su parte, la víctima estaba en condiciones de escuchar al victimario y de recibirle la luz entregada, con lo cual también estaba dispuesta a aceptar su solicitud de perdón, es decir, a perdonar.

Perdonar, en las circunstancias y dentro del padecimiento que ha conllevado para las víctimas el perder a sus seres más queridos y cercanos o tenerse que desplazar de su lugar de origen por la imposi- ción de alguien, no era algo fácil, pero llenaba de esperanza a toda la comunidad educativa. Así lo relata la señora Teresita Gaviria7, directora del programa Madres de la Candelaria:

Si bien al principio teníamos dudas por lo que generaba el compartir el mismo espacio con los excombatientes, el haber podido realizar encuentros de reconciliación con ellos fue muy importante; a partir de ahi se convirtió en un lugar muy bonito y agradable donde mis estudiantes se sentian muy contentas de poder estudiar, además de interactuar con toda la comunidad educativa.

Estos espacios eran acompañados por grupos de estudiantes que hacían parte del cepar. Por su gran significado como ejercicio de formación, indudablemente estas acciones movilizaban sentimientos que, a su vez, podían traducirse en nuevas formas de comportamiento, a partir del valor de la reconciliación como medio para respetarse a sí mismo y a los demás; también daban la posibilidad de hacer visible la importancia de la no repetición, de darle una mirada distinta como sociedad a partir de escenarios de sana convivencia, donde los estragos del conflicto armado deberían ser evitados con acciones de promoción de la no violencia.

\section{Inclusión escolar como escenario reparador}

Era muy importante que este contexto educativo, que acogía a estos grupos poblacionales, creara espacios de inclusión regidos por parámetros de equidad e igualdad, buscando siempre garantizar en los estudiantes un proceso de aprendizaje de calidad. Según Echeita y Ainscow (2011), la inclusión busca brindar respuestas apropiadas al amplio espectro de necesidades de aprendizaje, en entornos tanto formales como no formales de la educación. El CEPAR de ninguna manera podía ser ajeno a ello y, por el contrario, debía hacer un ejercicio mucho

7 Directora de Caminos de Esperanza Madres de la Candelaria. 
más riguroso en términos de inclusión, teniendo en cuenta que sus participantes habían estado privados buena parte de su vida del acceso a la educación e incluso algunos nunca pudieron acceder a una escuela cuando niños.

Se planteó, por lo tanto, acciones encaminadas al apoyo de estudiantes que presentaban dificultades de aprendizaje. La mayoría de ellos pertenecían a los grados de educación primaria. Se fueron identificando en muchos de ellos dificultades en los dispositivos básicos del aprendizaje, como la memoria, la atención, la percepción e incluso la motivación, lo cual generaba un reto mayor dentro del proceso de enseñanza-aprendizaje.

Ante esto se creó, por iniciativa de los mismos profesionales capacitadores y formadores, un grupo de estudio conformado por algunos de estos profesionales, en especial los que acompañaban los cursos de niveles iniciales (básica primaria). El propósito era analizar las dificultades de aprendizaje y pensar en estrategias para su abordaje, entendiendo la importancia que tiene la educación como una acción que no solo instrumentaliza sino que emancipa a sus estudiantes (Da Trindade y Santos, 2015). Este grupo de estudio sirvió de apoyo en la atención de estudiantes con dificultades de aprendizaje y además promovió en el equipo de trabajo una perspectiva de mejor comprensión para con los estudiantes.

Tanto víctimas como victimarios ya compartían los mismos espacios de clase, lo que implicaba que interactuaran de manera permanente dentro de la jornada educativa. Fue así como en el marco de la inclusión se promovía el trabajo cooperativo entre estudiantes. Así lo relata la docente Luisa Fernanda Ríos ${ }^{8}$, que hizo parte de este proceso en calidad de docente y como coordinadora del proyecto de inclusión CEPAR; "el aula se convirtió en un puente para que tanto víctimas como victimarios reflexio-

8 Docente y coordinadora del proyecto de inclusión escolar del CEPAR entre 2008 y el 2018. Entrevista virtual, 9 de junio de 2020 . naran sobre las formas y las maneras en que habían aprendido anteriormente y además la manera en que ahora se estaba dando dicho proceso".

Como adultos, tenían a su haber experiencias importantes previas, aunque empíricas, que de alguna manera eran la base para la adquisición de nuevos conocimientos, que se construyen sobre la base de concepciones, representaciones y conocimientos de sus experiencias de vida (Coll, 1991). Esto les permitía generar confianza dentro del aula, donde sus integrantes, tanto de un grupo como del otro, podían reconocer conjuntamente aspectos del aprendizaje, los cuales se convertían en el punto de partida para el trabajo mancomunado que se empezaba a gestar entre ellos. Así, las ideas y conceptos en torno a la educación iban ganando fuerza cada día y ya se empezaba a dejar atrás situaciones de vida atravesadas por el conflicto armado. Así lo refiere Luisa Fernanda Ríos: "el escenario de aprendizaje fue generando cambios para que el sentido que ellos tenían de la guerra y del conflicto, o las razones por las cuales muchos de ellos estuvieron ahí sumergidos, perdiera valor y sentido, construyendo así otros sentidos de vida, nuevos proyectos de vida".

Se registraron escenas donde, por ejemplo, una mujer víctima de aproximadamente 48 años de edad se acercaba donde uno de sus compañeros, quien era un victimario, y con gran paciencia le explicaba cómo hacer un ejercicio matemático. En este contexto del CEPAR, era muy importante que esto sucediera (reparación y reconciliación), ya que sus participantes habían estado atravesados por historias de vida difíciles donde incluso su compañero de estudio pudo ser perfectamente el perpetrador de un crimen y sin embargo estaba dispuesto a ayudarlo.

La mejor manera de explicar cómo la inclusión constituye un espacio de reparación es imaginando un aula de clase donde están presentes excombatientes de grupos de autodefensas y de grupos insurgentes de guerrilla, que habían sido enemigos en el conflicto armado y cuyo objetivo era eliminar 
a su oponente, y ahora se encuentran sin fusil en un mismo espacio de clase, sin más armas que un lápiz y un cuaderno; además, la escena incluye personas que fueron víctimas de las acciones que los dos grupos anteriores habían realizado. Pensemos, por lo tanto, en el nivel de tensión que dicha escena transmite, donde ya el contexto no es de guerra. Acá la educación resulta ser una herramienta poderosa capaz de contener y a su vez transformar el rencor, el miedo y el dolor en comprensión y solidaridad, y, por lo tanto, conducir a la reparación subjetiva.

Dentro del relato de la coordinadora de inclusión, Luisa Ríos, se describe una situación que da cuenta de esos aspectos, tanto de tensión como de superación a través de la educación inclusiva;

Hay una experiencia que recuerdo mucho y tiene que ver con un curso nuevo que se abrió para las madres de la Candelaria (víctimas) sobre tecnología. Se pretendia que ellas tuvieran un acercamiento a todo el tema relacionado con los computadores y se determinó que las personas que iban a brindar estos conocimientos básicos fueran un grupo de estudiantes más avanzados académicamente. Este grupo de estudiantes habian pertenecido a las autodefensas (victimarios), y durante el encuentro inicial ellos mismos le expresaron a las victimas quiénes eran o qué habian sido antes, pero la respuesta de este grupo de mujeres fue de aceptación $y$ en ningún momento se negaron a que estos excombatientes fueran los que les iban a dictar dicho curso, esto a pesar de los sentimientos encontrados que de parte de los desmovilizados se hallaban, donde, por un lado, se mostraban motivados y con un alto sentido de responsabilidad para con la tarea asignada, pero, por otro lado, con ciertos temores ante las posibles reacciones de desaprobación que aquellas personas, victimas, pudiera manifestar.

El acto de ayuda desinteresada se convertía en una muestra de que las heridas podrían ir sanando si existe un factor y un objetivo en comunes. Probablemente, aquella estudiante víctima que se acerca a su victimario para ofrecer su ayuda es la mejor manera de evidenciar que hay un proceso de reparación interno, en la medida en que ese victimario iba mostrando un deseo de aprender, superando los obstáculos, y emprendía una posibilidad de vida diferente.

\section{Convivencia como factor reconciliador}

La convivencia escolar es un proceso interrelacional con una dimensión interpersonal y colectiva (Fierro, 2013). El CEPAR debía ser garante de un aspecto tan importante como este, teniendo en cuenta que sus usuarios habían estado inmersos en situaciones de conflicto armado donde la visión del otro está atravesada por los desacuerdos ideológicos y políticos, según la posición y el tipo de grupo armado al cual pertenecieran los excombatientes. Además, el grupo poblacional de víctimas tenían a su haber razones suficientes para descalificar a los excombatientes por los actos cometidos, generadores de tanto dolor para miles de familias que habían padecido el rigor de la guerra; por lo tanto, se debía implementar un proceso que conllevara un ejercicio potente de sana convivencia dentro de las aulas de clase.

En Colombia, el Ministerio de Educación Nacional (MEN) es quien dicta las políticas en relación con los procesos de convivencia que se deben implementar en los centros de formación escolar, mediante la Ley 1620 de 2013, que "crea el Sistema Nacional de Convivencia Escolar y formación para el ejercicio de los Derechos Humanos, la Educación para la Sexualidad y la Prevención y Mitigación de la Violencia Escolar" (MEN, 2013). Si bien tanto esta como otras normativas establecen parámetros para que las instituciones educativas organicen sus manuales o pactos de convivencia, el CEPAR debía construir -a partir de la experiencia inédita que conllevaba la atención de población excombatiente y de víctimas del conflicto-, elementos y estrategias que fueran más allá de un ejercicio de convivencia sana. 
La convivencia se convirtió en un eje transversalizado que debía promoverse en todo momento. De acuerdo con lo referido por Lina Colorado9, quien hizo parte de este centro de formación como coordinadora de convivencia, siempre se presentó el interrogante sobre cómo lograr una sana convivencia entre personas tan distintas que en otro tiempo fueron enemigas. Al respecto, ella refiere: "Si bien este proceso ha proporcionado educación a una diversidad de personas a primera vista antagónicas, pero, si se revisan con detenimiento sus características, es evidente que hay condiciones en las que coinciden, y es allí donde se genera la posibilidad de la convivencia".

Estas coincidencias se pueden establecer a partir del deseo de poder vivir experiencias relacionadas con la formación escolar que en su momento no se dieron, porque no tuvieron dicha posibilidad o porque en sus propias decisiones hallaron formas de vida diferentes a la educación, pero que hoy encuentran una alternativa diferente con un objetivo en común para todos, y es el proceso de aprendizaje, que a su vez da posibilidades de construir un proyecto de vida diferente en ellos.

Otro aspecto en común entre víctimas y victimarios es que unos y otros habían vivido dificultades similares y pasado por experiencias dolorosas a causa de la violencia y la pobreza, muchos incluso habían sido expulsados del sistema educativo formal, por lo que encontraron en el CEPAR un lugar donde se cree en ellos, junto con el reconocimiento hacia el equipo docente y el personal de apoyo administrativo como figuras significativas que los valoraban como interlocutores, factores que fortalecen el sentido de pertenencia con el centro de formación y con su mismo proceso educativo. Lina Colorado describe el sentido de pertenencia como ligado a "un lugar de acogida y fácil acceso que ofrece recursos pedagógicos diferenciales para incrementar la permanencia y la inclusión, y el compromiso con

9 Psicóloga, coordinadora de convivencia CEPAR, 2011. un proceso de crecimiento personal que les permite proyectar metas a mediano y largo plazo realizables y socialmente aceptadas".

Algunas de las acciones concretas que se realizaban en el marco de la convivencia tenían que ver con:

Generar motivación: el primer contacto es fundamental para transformar la idea preconcebida que tienen muchos acerca de la dificultad para aprender. Aparece, por ejemplo, la idea de que va a ser muy difícil debido al alejamiento del sistema de educación durante buena parte de la vida. Generar motivación y confianza en los estudiantes puede incrementar la eficiencia no solo en el proceso de formación (Zapata Gómez y Rojas, 2010), sino también en la interacción con los demás. Así, para algunos estudiantes el hecho de no haber estudiado en su infancia hacía que sus mentes estuvieran muy "cerradas", lo cual los desmotivaba con el proceso.

Apertura, escucha y FLEXIBILIDAD: se destaca la atención permanente para los estudiantes a través del diálogo con sus docentes directivos y profesionales de apoyo administrativo, lo cual les generó la experiencia de ser escuchados, que su punto de vista es importante y que a través del diálogo se pueden resolver fácilmente los conflictos. También se destaca el hecho de que pudieron acceder a las diferentes jornadas programadas de acuerdo con sus necesidades, por ejemplo, podían asistir varios días a la semana, ya fuera en la jornada de la mañana, por la tarde o en la noche, pero además podían inscribirse para asistir un solo día incluyendo jornada dominical.

RACIONALIZACIÓN DE NORMA: la norma impacta tanto en la convivencia armónica y pacífica como en la motivación por aprender (Rodríguez, 2018). Este parámetro, directamente ligado a la convivencia, promovía desde un comienzo el conocimiento de las reglas de juego, su lógica y su justificación. Dichas normas eran socializadas amablemente y explicadas hasta que el estudiante reconocía su validez y la aceptaba. Esto debía ser un proceso formativo de todos los días, donde se demostraba que, si bien 
había un grado importante de flexibilidad también era necesario establecer algunos límites que les permitiera comprender y asimilar la necesidad del cumplimiento de las normas (García y Ferreira, 2005), procurando que se llegarán a acuerdos y que estos se respetaran. Para ello, se constituyó un comité de convivencia, como instancia encargada de analizar las variables que intervenían en los procesos de convivencia del CEPAR.

La sana convivencia en el centro de formación era una premisa fundamental y punto de partida para que los participantes también la trasladaran a otros escenarios de vida, en los ámbitos familiar, laboral y comunitario, con el fin de garantizar una paz más allá de un pacto o un acuerdo; es decir, que se trascendiera a una paz positiva, que va más allá de la ausencia de violencia o de guerra y procura la integración de la sociedad humana, algo así como "un patrón de cooperación e integración entre los principales grupos humanos", tal como lo refiere Galtung (1964, citado en Harto, 2016, p. 131).

En el tema puntual de la paz, se realizó un trabajo con el apoyo de la Fundación Prem Rawat ${ }^{10}$, coordinado por el área psicosocial del CEPAR, donde se planeó, conjuntamente con el componente psicosocial, el área de ética y valores, y un trabajo conjunto denominado Educación para la Paz, con temáticas diversas sobre paz, realidad social, política, conflicto, entre otros temas. Además, se desarrolló un trabajó más profundo enfocado en la "paz interior" que tenía como propósito ayudar a cada participante a descubrir los recursos más valiosos: la fuerza interior, la capacidad de elección, la esperanza y la posibilidad de sentir paz, y hacer de ellos un instrumento práctico en su vida; como lo describe Rawat: "la paz está justo dentro de nosotros, pero muchas personas nunca llegan a experimentarla" (2020).

10 La Fundación Prem Rawat (TPRF The Prem Rawat Foundation) ofrece ayuda social y cursos de Educación para la Paz, que han tenido una gran repercusión en centros de estudio de todo nivel, prisiones, veteranos de guerra y asociaciones profesionales en más de setenta países, en los seis continentes, traducidos a 36 idiomas.
Se destaca de este trabajo la selección de un grupo focal compuesto por excombatientes y víctimas del conflicto que permitió fortalecer la reconciliación, elaborando y despertando en la subjetividad la posibilidad de paz interior y proyectándola hacia los otros. Tal como lo expresa la señora Martha Elena Pineda", en Educación para la Paz "tienen la oportunidad de explorar sus recursos internos y utilizarlos como herramientas para que su vida pueda transformarse. El programa busca una transformación en los valores de las personas para que puedan encontrar su paz y ayudar así a construir la paz colectiva".

Destaco a continuación algunos comentarios de dos estudiantes ${ }^{12}$ que participaron en el grupo focal sobre la paz, que dan cuenta del trabajo ahí realizado y su impacto personal. El primero corresponde a un excombatiente masculino de aproximadamente 22 años de edad: "con el Programa de Educación para la Paz por primera vez sentí que podía dejar atrás mi pasado, podía soltar mi carga, vivir en el ahora, sentir paz. El programa me ayudó a cambiar, a transformar mi ser en una persona que me gustaba más, empecé a dejar mis hábitos de reaccionar mal ante los demás". El segundo estudiante es una excombatiente de aproximadamente 28 años de edad:

Me di cuenta que, aunque a veces vivimos lo que nos tocó, no lo que elegimos, por difícily duro que haya sido, uno siempre puede cambiar. Empecé a sentir que mi pasado quedaba atrás. No me avergüenzo de mi historia y a mi hija ya le estoy infundiendo todo lo que yo he aprendido con el Programa de Educación para la Paz, que todo está en nosotros y que la ira y los rencores no sirven.

11 Además de hacer parte del equipo de voluntarios, sirve de enlace entre el equipo de voluntarios para Colombia del programa Educación para la Paz y la Fundación PremRawat. Entrevista por videollamada, enero de 2020.

12 Los comentarios registrados hacen parte de los relatos de algunos estudiantes participantes en el programa Educación para la Paz llevado a cabo en el CEPAR y han sido recopilados por el equipo de voluntarios que realizaron dicho proceso. La información fue facilitada por la señora Martha Elena Pineda. 


\section{Acompañamiento psicosocial como elemento integrador}

El trauma psicosocial experimentado por las personas denota unas relaciones sociales enajenantes (Baró, 1989); de ahí la importancia de un proceso de atención psicológica. Los excombatientes contaban con el apoyo y acompañamiento por parte de un equipo psicosocial en el marco del proceso de desmovilización que ayudaba a su adaptación a la ruta educativa que emprendían en el CEPAR. También las víctimas recibieron apoyo psicosocial por parte del programa de víctimas perteneciente a la alcaldía de Medellín, con el fin de procurar un buen proceso de adaptación dentro de este espacio educativo.

La atención psicosocial se entiende como el acompañamiento e intervención directa que ofrecen los profesionales tanto a los desmovilizados como a las víctimas del conflicto. De acuerdo con Back (1963) y Torregrosa (1974) -citados por Ibáñez (1990)-, el acompañamiento psicosocial se refiere a las prácticas basadas en la relación entre individuo y sociedad. Por otro lado, por psicosocial se entiende la generación de conocimientos multidisciplinares y diversos que se conectan entre sí.

Cuando se dieron los primeros encuentros de reconciliación dentro del CEPAR entre víctimas y victimarios, el acompañamiento psicosocial fue un factor preponderante para que este ejercicio se pudiera dar en las circunstancias que se necesitaba. Los estudiantes, por lo tanto, sentían el respaldo de los profesionales, que les ayudaban a generar estrategias para afrontar potenciales situaciones difíciles de abordar por sí solos.

La intervención psicosocial logró establecer un apoyo transversal a toda la ruta educativa del CEPAR. Dicha área daba acompañamiento al proyecto de inclusión educativa por medio de valoraciones psicológicas a estudiantes con dificultades de aprendizaje, además de espacios de intervención individual y grupal. Así mismo, un profesional psicosocial acompañaba al comité de convivencia escolar, aportando a las decisiones que se tomaban en relación con las dificultades de interacción que se podían presentar con los estudiantes. Cuando se evidenciaba la necesidad de una intervención individual psicológica, se asignaban unos espacios de asesoría que aportaban al mejoramiento del comportamiento del estudiante y a la convivencia en general; además, se daba apoyo el consejo académico en lo relacionado con la construcción de currículos acordes a las necesidades de los estudiantes.

Tanto los desmovilizados del conflicto como las víctimas presentaban situaciones de orden psicoemocional que se debía intervenir teniendo en cuenta las particularidades de cada caso, procurando con ello el fortalecimiento de sus recursos personales psicológicos, de modo que pudieran instaurar nuevos lazos asociativos para asimilar subjetivamente los fenómenos sociales adversos (Ansermet y Mejía, 1999, como se citó en Aristizábal et al., 2012) y reparar aquellos aspectos traumatizantes que han acompañado a estos estudiantes en buena parte de sus vidas, es decir, resolver sus conflictos internos y así adaptarse más adecuadamente a las circunstancias de su medio (Coderch, 2012), además de promover la resignificación de un nuevo proyecto de vida personal.

Se describen a continuación las formas de intervención psicosocial que se activaron en el proceso como apoyo a víctimas y victimarios:

INTERVENCIÓN INDIVIDUAL: las situaciones difíciles que a lo largo de la vida han atravesado la mayoría de los usuarios del CEPAR van determinando la necesidad fundamental de su abordaje a través de la atención psicológica, aportando a su proceso de transformación personal. Tales circunstancias están determinadas por características propias de una población que, ante los cambios o la permanencia de situaciones adversas, son más vulnerables (Busso, 2001); por ejemplo, la deprivación escolar en las primeras etapas de desarrollo, la desmotivación frente a las actividades educativas, estudiantes en extra 
edad escolar para primaria y bachillerato por vinculación a los grupos armados, entre otras.

Otro factor importante a tener en cuenta es la historia familiar que acompaña la biografía tanto de víctimas como de victimarios y que ha estado atravesada por múltiples dificultades, que incluyen abandonos, agresiones, abusos, entre otras, siendo esto un predictor de problemas emocionales y sociales (Di Bártolo, 2016), muchos de los cuales tienden a repetirse en comportamientos y actitudes, que en su mayoría son disfunciones a la hora de establecer vínculos de todo orden con los demás. De igual forma, el no tener la posibilidad de enfrentar sus adversidades, ya sea por los escasos recursos psicológicos propios o por no contar con un apoyo externo que los oriente acerca de la manera en que se pueden asumir las adversidades, les generaba crisis personales, deserción académica o laboral, relaciones interpersonales inadecuadas o escasas e incluso el no avance en su proceso educativo. En tal sentido, la atención psicológica individual brindaba a los estudiantes de CEPAR diversas formas de intervención: en crisis, asesorías y psicoterapia (breve).

De acuerdo con Hernández y Gutiérrez (2014), la intervención en crisis consiste en un tratamiento intensivo que requiere la habilidad para la creación de un clima óptimo de comunicación que facilite: la libertad de expresión por parte de la persona en crisis, la aceptación de la persona y de su situación, la expresión de la capacidad para escuchar de manera empática, la búsqueda de soluciones realistas y la expresión natural de afecto y cercanía.

Dadas las circunstancias y situaciones de vida de los desmovilizados del conflicto y de las víctimas, era frecuente que algunos presentaran alteración psicoemocional, que irrumpía inesperadamente durante la jornada académica a la cual asistían; por lo tanto, se debía hacer contención emocional, además de propiciar una estabilidad psicológica que garantizara las condiciones para retomar las actividades académicas.
En una ocasión, a una mujer víctima del conflicto, de aproximadamente 54 años de edad, cuya crisis de ansiedad le impedía estar en el aula de clase, una vez estabilizada relataba lo siguiente: "yo no sé qué me pasa, que como que no me concentro; me sucede que cuando estoy en clase a veces llegan unos pensamientos a mi mente que no me dejan concentrarme (entra en llanto), me acuerdo de cuando mataron a mi esposo y a mis tres hijos por allá donde vivíamos".

Con frecuencia se debían ofrecer espacios de orientación y asesoría psicológica a los estudiantes, quienes en su mayoría lo solicitaban de forma voluntaria, o en otros momentos eran derivados por los mismos docentes o por otras áreas, como la de convivencia o la de inclusión educativa. Se tenía establecido, aunque no de manera rigurosa, que para cada estudiante se ofrecían hasta seis sesiones de orientación psicológica, esto con el fin de garantizar la atención de todos aquellos que demandaban dicha asesoría.

En algunos casos puntuales, se establecía un acompañamiento más extenso, constituyéndose en un proceso de psicoterapia breve, buscando una planificación de objetivos terapéuticos de manera sistemática en un tiempo limitado (Ávila Rojí y Saúl, 2011), ya que las circunstancias así lo requerían, avalado además por el mismo usuario. Este fue el caso de una excombatiente, de aproximadamente 26 años de edad, que requería un abordaje más extenso debido a traumas desarrollados desde su infancia: "yo nunca he tenido quién me aceptara; desde pequeña siempre fui rechazada, e incluso cuando tenía como 12 años un hermano abusó de mí y me amenazó que si contaba me mataba, y me fui para la guerrilla".

Según Coderch (2012), los traumas indefectiblemente disminuyen la capacidad en las personas para manejar los sentimientos y las ideas, dado que lo sienten todo como demasiado real en relación con los acontecimientos internos. Por lo tanto, es necesario, dentro de este ámbito educativo, facilitar a 
estos estudiantes la posibilidad de tener espacios de atención y contención, propiciando estabilidad para que puedan tener una mejor disposición frente al proceso de aprendizaje.

INTERVENCIÓN GRUPAL: El espacio grupal tiene un gran valor como reconstitución del sentido de las experiencias colectivas (Baz, 2007). El trabajo grupal dentro del CEPAR proponía intervenciones centradas en la ayuda mutua, desarrollo de la solidaridad, participación ciudadana, competencia para el análisis de situaciones y toma de decisiones que afectan a un colectivo, habilidades de trabajo en equipo, etc.

Algunas temáticas y actividades que se desarrollaron durante este proceso formativo y con los parámetros del acompañamiento psicosocial grupal estuvieron pautados por: 1) la orientación vocacional, mediante la ayuda a los estudiantes a conocerse a sí mismos, conocer las posibilidades del entorno, adquirir habilidades en la toma de decisiones (Bisquerra, 2012); 2) la realización de campañas pedagógicas de prevención del consumo de sustancias psicoactivas, cultura de la legalidad, sexualidad segura, medio ambiente; 3) el apoyo al proyecto de inclusión educativa con estimulación cognitiva, promoción de motivación escolar (abordando situaciones de orden subjetivo que generen en los estudiantes problemáticas emocionales y que lleven a que renuncien con facilidad a los procesos); y 4) acciones de promoción de la salud mental de acuerdo con los parámetros de la Organización Mundial de la Salud (OMS) en lo relacionado con las habilidades para la vida (Martínez, 2014).

A modo de ejemplo de intervención psicosocial, relato lo siguiente: en una ocasión, en un grupo de niveles iniciales (tercero de primaria), en el que participaban tanto excombatientes como víctimas, se dialogaba acerca de la posibilidad de un proceso de paz con la guerrilla de las Fuerzas Armadas Revolucionarias de Colombia (Farc) que llevaría a que algunos de sus integrantes pudiesen participar en política y se les asignaran algunas curules en el Congreso de la República. Una de las víctimas, mujer de aproximadamente 60 años de edad, se exaltó mostrando su inconformismo y desacuerdo con las prebendas que se proponían ante aquel posible acuerdo de paz, y expresaba su indignación con palabras ofensivas dirigidas hacia dicho grupo armado, sin pensar ni reflexionar que en el aula de clase había excombatiente del grupo insurgente. Esto hizo que uno de ellos se sintiera aludido y decidiera acudir al área psicosocial para hablar de la situación. En dicho espacio, el estudiante logró expresar los sentimientos que lo desestabilizaban emocionalmente hasta que, ya más tranquilo, retomó las actividades académicas.

Dentro de la intervención realizada en este caso, se practicó una atención en crisis donde, además, se garantizó un abordaje con la contraparte recalcando la importancia de manejar de manera prudente las concepciones personales en relación con temas que pueden generar en las otras personas discrepancias, máxime cuando las circunstancias y las personas inmersas en este centro formativo han padecido durante buena parte de su vida situaciones difíciles que tal vez quieran dejar atrás.

El abordaje de la situación anterior conllevo realizar una intervención con la mujer víctima del conflicto que había hecho el comentario, donde pudo comprender que era necesario ubicarse en el contexto, ya que sus palabras podían abrir heridas $y$, al igual que sus propias heridas, exacerbar los ánimos, hasta detonar posibles conflictos interpersonales. En el espacio de orientación psicológica se le hizo claridad a ella que su sentimiento era válido desde su punto de vista como víctima, pero que, a su vez, era necesario que, dentro de este espacio de formación, se diera un diálogo regido por parámetros diferentes al de las expresiones agresivas. También se realizó una intervención grupal en el aula, donde se presentaron los hechos, con el fin de reflexionar de manera conjunta en los aspectos acerca de los cuales se había dado la discusión. El grupo lo asumió de una manera tranquila, pero consciente de la necesidad del respeto por las ideas del otro 
y de la importancia de entablar una comunicación más racional y menos impulsiva.

Antes de concluir, es necesario entender que en este tipo de procesos se hallarán obstáculos, y esta no fue la excepción, pues hubo momentos de tensión. Por ejemplo, como lo relata uno de los excombatientes de las autodefensas, luego de haber culminado con éxito su proceso de formación profesional como ingeniero, recibió la oportunidad de vincularse como capacitador y docente en el CEPAR, y ya en su rol de docente relata la siguiente situación:

Cuando hacia el año 2007 inicio mi proceso como docente en el CEPAR, recuerdo que el coordinador me presento a un grupo, el cual eran victimas del conflicto, personas muy mayores de edad, y evidentemente al yo ser desmovilizado, no importaba que ya fuera el educador, me vi reflejado como una persona mala, recordando una cantidad de cosas, al ver que todas estas personas eran víctimas; además se les comunicó que yo había sido desmovilizado, y no mostraron buena disposición al enterarse de ello, e incluso recuerdo que la mayoría se retiró del salón; quedé, por lo tanto, con algunos pocos en el aula de clase, con los cuales pude hablar. Me presenté con mucho respeto, [y] ante la situación no desistí, lo asumi como un reto personal tratar de encajar ahí. En la siguiente clase retornaron las personas que se habian ausentado por mi presencia y por mi calidad de excombatiente. Tal vez pensaban que habian cambiado el docente, que yo ya no estaba ahi; sin embargo, con un trabajo de diálogo, de concertación, y yo como docente debía tener un manejo y un control de la situación, pero lo hacía con mucho respeto. Además admiraba y les reconocía a ellos los logros que alcanzaban en el aprendizaje animándolos con ello a continuar. De ahí sentí que ya era aceptado por las víctimas, debido al trabajo que se iba consolidando cada día y donde ellas mismas incluso recomendaban a sus compañeras victimas para que se vincularan al CEPAR, ya que encontraban un espacio favorecedor para su proceso educativo. Considero, por lo tanto, que fue a través de la educación que fue posible que se diera este evento de reconciliación. Fue una experiencia muy bonita para mí, de la cual me siento muy feliz, por haber logrado captar la atención que quería en ellas para ayudarles y acompañarlos en su proceso.

\section{Conclusiones}

Una de las conclusiones es que la educación es una herramienta poderosa, en procesos tanto de adquisición de conocimiento como de transformación social, que contribuyan de manera decisiva a formar personas que aprendan a pensar por sí mismas pensando en los demás (Jover, 2013). El CEPAR ha sido un claro ejemplo de que se pueden brindar posibilidades de vida diferentes a personas en condiciones de vulnerabilidad marcadas por los rigores del conflicto y de la guerra en nuestro país. Sin lugar a dudas, se hace necesario redireccionar los procesos en búsqueda de opciones de vida con un contenido de reparación y de reconciliación interior, como víctima o victimario del conflicto armado.

Es importante destacar las posibilidades que brindan las acciones de intervención psicosocial en el ámbito educativo, máxime en procesos donde los participantes y comunidades se encuentran en condiciones adversas de vulnerabilidad psicoafectiva. Si bien las instituciones educativas tienen una función primordial enfocada al quehacer pedagógico, es evidente que la sociedad actual -con las complejas dinámicas entre sus actores y los contextos diversos en que estos se mueven- se encuentra afectada por diferentes problemáticas psicosociales, que justifican sin duda la labor comprometida de la psicología. Para ello, el desarrollo científico y práctico de la psicología ha permitido que el reconocimiento de los diferentes procesos cognitivos, socioemocionales y culturales oriente las acciones que aportan al bienestar de los sujetos, los grupos sociales y las comunidades en general (Vera, 2018). 
En Colombia, al igual que en muchos países del mundo, hay cada vez mayor apertura a otras disciplinas dentro de la educación, entendida como sistema, y dentro de ellas la psicología, en su interacción con la educación, ofrece varias posibilidades, como las que se vieron reflejadas en este texto, que aportan no solo a la formación sino a la transformación de las historias de vida. Destaco algunas de ellas de manera puntual:

La inclusión escolar, que busca detectar, evaluar e intervenir en aquellas situaciones que interfieran en el normal desarrollo del aprendizaje y que se hacen más evidentes en el trabajo con personas adultas o en extra edad escolar. Es necesario, por lo tanto, establecer acciones encaminadas a la inclusión, especialmente si se trabaja con población adulta, ya que tendría impacto en prácticamente todas las dimensiones de calidad de vida, particularmente en inclusión social, pero también en derechos, autodeterminación, desarrollo personal y relaciones interpersonales (López et al., 2009).

La convivencia escolar, que propende por el mejoramiento del clima escolar, la sana convivencia, la promoción de valores, entre otros, reflejo de la rele- vancia mundial que tienen la convivencia y la no violencia en la educación (López, 2014). Si algo ha sido realmente difícil de sobrellevar para los estudiantes que han pertenecido al centro de formación CEPAR han sido las dificultades vividas a lo largo de sus vidas, por culpa de la violencia, donde la convivencia pacífica ha estado ausente casi todo el tiempo; por ende, es imprescindible la intervención psicosocial en este sentido también.

Finalmente, las intervenciones psicosociales que se realicen, dentro del escenario educativo, ya sean de carácter individual o grupales, pueden y deben plantearse a una escala que va desde la prevención hasta la promoción o la intervención, de acuerdo con las necesidades y características de la población que se atiende. Atender población en condiciones de vulnerabilidad psicosocial como los participantes del proyecto CEPAR, que han sido víctimas del conflicto armado, o participantes de grupos armados (victimarios), conlleva cuidados en el abordaje, y para ello debe siempre coexistir un diálogo abierto disciplinar; en este caso, entre pedagogía y psicología, buscando que las acciones sean conjuntas y, por lo tanto, coherentes con la realidad de las estudiantes.

\section{Referencias}

Alonso, M. y Valencia, G. (2008). Balance del proceso de desmovilización, desarme y reinserción de los bloques Cacique Nutibara y Héroes de Granada de la ciudad de Medellín. Estudios Políticos, 33, 11-34. Recuperado de: http://www.scielo.org.co/scielo.php?pid=S0121-51672008000200002\&script=sci_arttext

Aristizábal, E., et al. (2012). Síntomas y traumatismo psíquico en víctimas y victimarios del conflicto armado en el Caribe colombiano. Psicología desde el Caribe, 29(1), 123-152. Recuperado de: https://www.redalyc.org/ pdf/213/21323171008.pdf

Ansermet, F. y Mejía, C. (1999). Trauma y lenguaje. Notas para una metodología de investigación clínica. En Servicio Universitario de Psiquiatría para Niños y Adolescentes (sUPEA), psiquiatría pediátrica anexa al CHUV, Hospital Nestlé, $\mathrm{CH}-1011$ Lausanne-CHUV. Francia. $\mathrm{N}^{\circ} 47,30$.

Ávila, A., Rojí, B. y Saúl, L. (2011). Introducción a los tratamientos psicodinámicos. Lerko. 
Baró, M. (1989). La violencia política y la guerra como causas del trauma psicosocial en El Salvador. Revista Psicológica de El Salvador, 7, 28, 123-141. Recuperado de: https://www.uca.edu.sv/coleccion-digital-IMB/ wp-content/uploads/2015/11/1988-La-violencia-pol\%c3\%adtica-y-la-guerra-como-causas-del-traumaRP1988-7-28-123_141.pdf

Baz, M. (2007). La intervención grupal: finalidades y perspectivas para la investigación. Área 3. Cuadernos de Temas Grupales e Institucionales, 11 (primavera). Recuperado de: http://www.areaz.org.es/sp/item/141/M.\%20 Baz:\%2OLa\%2ointervenci\%C3\%B3n\%2ogrupal:\%20finalidades\%20y\%2operspectivas\%2opara\%20la\%20 investigaci\%C3\%B3n

Bisquerra, R. (2012). Orientación, tutoría y educación emocional. Síntesis.

Bolaños, T. y Biel, I. (2019). La justicia transicional como proceso de transformación hacia la paz. Revista Derecho PUCP, 83, 415-442. Recuperado de: http://www.scielo.org.pe/pdf/derecho/n83/a14n83.pdf

Busso, G. (2001). Vulnerabilidad social: Nociones e implicancias de políticas para Latinoamérica a inicios del siglo XXI. [Ponencia] Seminario Internacional Las diferentes expresiones de la Vulnerabilidad Social en América Latina y el Caribe, Naciones Unidas, Santiago de Chile. Recuperado de: http://www.derechoshumanos. unlp.edu.ar/assets/files/documentos/vulnerabilidad-social-nociones-e-implicancias-de-politicas-paralatinoamerica-a-inicios-del-siglo-xxi.pdf

Casullo, M. M. (2005). La capacidad para perdonar desde una perspectiva psicológica. Revista de Psicología, 23(1), 39-63. Recuperado de: https://www.redalyc.org/articulo.oa?id=337829529002

Cendales, L. y Torres, A. (2006). La sistematización como experiencia investigativa y formativa. La Piragua, 23. Recuperado de: http://www.cepalforja.org/sistem/bvirtual/?p=710

Centro Nacional de Memoria Histórica (2015). Rearmados y reintegrados. Panorama posacuerdos con las AUC. Recuperado de: http://www.centrodememoriahistorica.gov.co/descargas/informes2015/desmovilizacionDesarmeReintegracion/desmovilizacion-y-reintegracion-paramilitar.pdf

Coderch, J. (2012). Realidad, interacción y cambio psíquico. Ágora Relacional.

Coll, C. (1991). Psicología y currículum. Paidós.

Cortés, C., Colorado, L., Serna, M. y Restrepo, M. (2011). Centro de formación para la paz y la reconciliación CEPAR: Un aporte a la paz desde la educación y la convivencia. Programa Paz y Reconciliación, Secretaría de Gobierno Alcaldía de Medellín.

Da Trindade. E. y Santos, A. (2015). Educación y aprendizaje a lo largo de la vida: los adultos y la enseñanza superior. Sintética, 45. 1-20. Recuperado de: https://sinectica.iteso.mx/index.php/SINECTICA/article/view/583

Di Bártolo, I. (2016). El apego: cómo nuestros vínculos nos hacen quienes somos. Lugar Editorial.

Echeita, G. y Ainscow, M. (2011). La educación inclusiva como derecho. Marco de referencia y pautas de acción para el desarrollo de una revolución pendiente. Tejuelo. Revista de Didáctica de la Lengua y la Literatura, 12, 26-46. Recuperado de: http://hdl.handle.net/10486/661330 
Fierro, M. (2013). Convivencia inclusiva y democrática. Una perspectiva para gestionar la seguridad escolar. Sintética, 40, 1-18. Recuperado de: https://sinectica.iteso.mx/index.php/SINECTICA/article/view/47

García Correa, A., Ferreira Cristofolini, G. (2005). La convivencia escolar en las aulas. International Journal of Developmental and Educational Psychology, 2(1), 163-183. Recuperado de: https://www.redalyc.org/ pdf/3498/349832309012.pdf

Hand, H. (2003). The mentor's tale: a reflexive account of semi-structured interviews. Nurse Researcher, 10(3), 15-2. DOI: http://doi.org/10.7748/nr2003.04.10.3.15.c5893

Harto, de V. (2016). La construcción del concepto de paz: paz negativa, paz positiva y paz imperfecta. Cuadernos de Estrategia, 183, 119-146. Recuperado de: https://dialnet.unirioja.es/servlet/articulo?codigo=5832796

Hernández, I. y Gutiérrez, L. (2014). Manual básico de primeros auxilios psicológicos. Universidad de Guadalajara. Centro Universitario de Ciencias de la Salud. Recuperado de: https://psicologosemergenciasbaleares.files. wordpress.com/2016/o8/manual-primeros-auxilios-psicolc3b3gicos_2014.pdf

Ibáñez, T. (1990). Aproximaciones a la Psicología Social. Asendai.

Jover, D. (2013). El poder de la educación para transformar la sociedad. Cambio Social y Cooperación en el Siglo XXI, 2, 82-94. Recuperado de: http://biblio3.url.edu.gt/PubliED/CambioSyC/6-Jover.pdf

López, D., et al. (2009). Proyecto INCLURED: inclusión en la escuela de adultos. Revista Española sobre Discapacidad Intelectual, 4O(2), 22-37. Recuperado de: https://sid.usal.es/idocs/F8/ART19428/david_lopez.pdf

López, V. (2014). Convivencia escolar. Apuntes. Educación y Desarrollo Post-2015, 4. Recuperado de: https://repasopcmasumet.files.wordpress.com/2018/og/convivencia-escolar-unesco1.pdf

Martínez, V. (2014). Habilidades para la vida: una propuesta de formación humana. Itinerario Educativo, 28(63), 61-89. DOI: https://doi.org/10.21500/01212753.1488

MEN - Ministerio de Educación Nacional (2013). Ley No. 1620 "Por la cual se crea el sistema nacional de convivencia escolar y formación para el ejercicio de los derechos humanos, la educación para la sexualidad y la prevención y mitigación de la violencia escolar". Recuperado de: https://www.mineducacion.gov.co/1759/ articles-327397_archivo_pdf_proyecto_decreto.pdf

Noguchi, F., Guevara, J. y Yorozu, R. (2018). Aprendiendo a lo largo de toda la vida para el desarrollo sostenible. unEsco. Recuperado de: https://unesdoc.unesco.org/ark:/48223/pfoooo265174

ODDR - Observatorio de Procesos de Desarme, Desmovilización y Reintegración (2010). Los procesos de desarme, desmovilización y reintegración: buenas prácticas y retos. Universidad Nacional de Colombia. Recuperado de: http://www.humanas.unal.edu.co/observapazyconflicto/files/4414/3584/9809/ODDR_Buenas_practicas_y_ retos_mayo_2010.pdf

PNUD - Programa de Naciones Unidas para el Desarrollo (2006). Modelo de intervención, regreso a la legalidad. Buenas prácticas para superar el conflicto. 
Rawat, P. (2020). Escúchate. Encuentra la paz en un mundo ruidoso. Nomos.

Rodríguez, A. G. (2018). Construcción de normas: una experiencia desde el clima de aula. Eleuthera, 18, 13-30. Recuperado de: http://www.scielo.org.co/pdf/eleut/v18/2011-4532-eleut-18-00013.pdf

UNESCO (2015). Educación 2030. Declaración de Incheon. Hacia una educación inclusiva, equitativa y de calidad y un aprendizaje a lo largo de la vida para todos. Recuperado de: http://www.unesco.org/new/fileadmin/ MULTIMEDIA/FIELD/Santiago/pdf/ESP-Marco-de-Accion-E2O3O-aprobado.pdf

Vera, M. (2018). Comprensiones y reflexiones sobre la acción psicosocial en el contexto educativo. Documentos de Trabajo ECSAH, 2, 2-3. Recuperado de: https://doi.org/10.22490/ECSAH.2847

Zapata, C., Gómez, M. y Rojas, M. (2010). Modelado de la relación de confianza profesor-estudiante en la docencia universitaria. Educación y Educadores, 13(1), 77-90. Recuperado de: http://www.scielo.org.co/pdf/eded/ v13n1/v13n1ao6.pdf 
